\title{
Collaborative Actions to Enable Richer and More Complex Planetary Science Mission Data
}

\section{Authors:}

\author{
T. Joseph W. Lazio \\ Jet Propulsion Laboratory, California Institute of Technology \\ 818-354-4198 \\ Joseph.Lazio@jpl.nasa.gov \\ Sami Asmar (Jet Propulsion Laboratory, California Institute of Technology) \\ Francisco Córdova (Arecibo Observatory, Univ. Central Florida)
}

E. J. Murphy (next-generation Very Large Array, National Radio Astronomy Observatory)

\author{
Endorsing: \\ David H. Atkinson (Jet Propulsion Laboratory, California Institute of Technology) \\ Nancy Chabot (Johns Hopkins University Applied Physics Laboratory) \\ Ian J. Cohen (Johns Hopkins University Applied Physics Laboratory) \\ Noam R. Izenberg (Johns Hopkins Applied Physics Laboratories) \\ Thomas K. Kim (Los Alamos National Laboratory) \\ Glenn Orton (Jet Propulsion Laboratory, California Institute of Technology) \\ Mark Hofstadter (Jet Propulsion Laboratory, California Institute of Technology)
}

Part of this research was carried out at the Jet Propulsion Laboratory, California Institute of Technology, under a contract with the National Aeronautics and Space Administration. The National Radio Astronomy Observatory is a facility of the National Science Foundation operated under cooperative agreement by Associated Universities, Inc. Some of the information presented in this white paper is pre-decisional and is for discussion and planning purposes only. 


\section{Introduction}

The science return from a mission is dictated ultimately by the quantity and quality of science data obtained. As only one recent example, following the initial return of data from the 2019 January flyby of the New Horizons spacecraft past (486958) Arrokoth, an improved understanding of its geology, geophysics, composition, and origin has been obtained as additional data have been returned from the spacecraft (Grundy et al. 2020; McKinnon et al. 2020; Spencer et al. 2020). Additional data are expected to be returned from the spacecraft yet in 2020, with the expectation that further refinements and discoveries are possible about this Kuiper Belt Object. We stress that New Horizons at Arrokoth is only one such example; similar experience has been observed for the Mars Reconnaissance Orbiter (MRO) mission and many others.

This white paper focuses on potential collaborative activities between NASA and NSF, with a focus on NASA's Deep Space Network (DSN) and NSF radio astronomical telescopes in order to enable larger data volumes to be obtained from spacecraft. Notable examples of such collaboration include the Very Large Array (VLA) in enabling the reception of data from Voyager 2's flyby of Neptune (Figure 1, Layland \& Brown 1985; Rogstad, Mileant, \& Pham 2003) and the use of the Green Bank Telescope (GBT) to track the descent of the Huygens probe to the surface of Titan and enabling the Doppler Wind Experiment (Folkner et al. 2006). Another example of collaborative work between NASA and the NSF to advance Planetary Science and Astrobiology, covered in many other white papers, is the ground-based planetary radar observations conducted with NASA's DSN and the NSF's Arecibo Observatory and GBT.

More generally, this collaboration extends internationally, dating back to at least the Voyager 2 encounter at Uranus, when the $70 \mathrm{~m}$ antenna at the Canberra Deep Space Communications Complex was paired with the $64 \mathrm{~m}$ Parkes Radio Telescope.

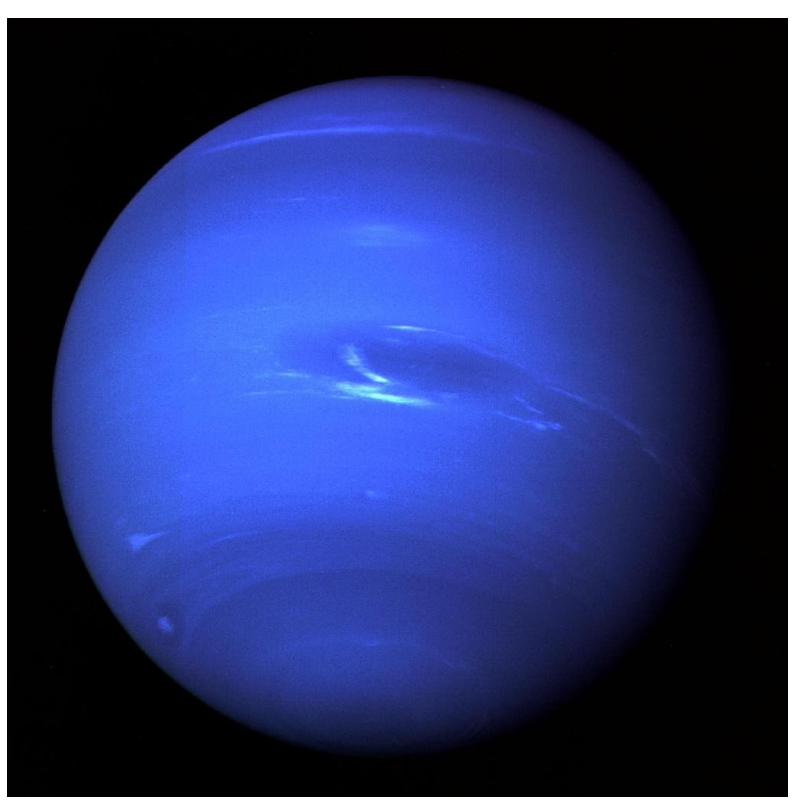

Figure 1: Neptune as viewed by Voyager 2, on 1989 August 20, just under five days from closest approach. The data for this image were downloaded via a combination of telemetry downlinks from the Very Large Array (VLA) and NASA's Deep Space Network (DSN). In a similar manner, future collaborative activities using existing and future NASA and NSF facilities could enable more ambitious Planetary Science missions. 


\section{Scientific Context}

Historically, NASA's most demanding application for highly sensitive ground receiving stations has been Planetary Science missions, often at distances of multiple astronomical units. Increasingly, Heliophysics missions have begun to demand similarly sensitive ground receiving stations. The most striking and cross-cutting such example is the Voyager Interstellar Mission, now considered a Heliophysics mission as it explores beyond the heliopause, but that started as a Planetary Science mission.

We now illustrate mission concepts or science investigations that could motivate such collaborative interactions between NASA's DSN and NSF radio astronomical facilities. This listing is intended to be illustrative only and is certainly not exhaustive. Nonetheless, a common theme is ensuring robust and large data volume returns for short-lived events or short-duration missions.

Venus concepts There is considerable interest in the Venus community in a surface lander in order to understand the habitability of Venus in part by determining the mineralogy and the composition of the rocks, such as was described in the New Frontiers-class Venus In Situ Explorer (VISE) concept (Visions 6 Voyages). The Venus Flagship Mission concept, under the Planetary Mission Concept Studies (PMCS) program, repeats the call for a surface asset. While material properties and thermal control systems have improved since the Soviet Venera landers, the surface of Venus is such a harsh environment that a state-of-the-art lander still will have a lifetime of only about $7 \mathrm{hr}$.

Ice Giant concepts The Ice Giants Pre-Decadal Survey Mission Study Report considers flagship-class mission concepts to Uranus, Neptune, or both. Four mission architectures were studied in detail, with three of the four including an atmospheric probe in order to sample atmospheric composition. (Two architectures involved an atmospheric probe for Uranus, one for Neptune.) The Neptune Odyssey concept in the PMCS program also carries an atmospheric probe. A probe in the atmosphere of either planet has an expected lifetime of approximately $1 \mathbf{~ h r}$. More generally, approximately a dozen white papers were submitted describing concepts for ice giant missions or Ocean Worlds studies at Uranian or Neptunian moons.

Mercury Lander The Mercury Lander is a PMCS concept that would conduct in situ surface measurements. The lander would rely on direct-to-Earth communications, and hence returning the full data volume before the lander suffers thermal failure due to the intense solar isolation is required. In order to meet this requirement, the concept study was not able to return the full highest frequency data set for the landed accelerometer payload, reducing the ability to measure seismic events on Mercury over the course of the landed mission.

Saturn Probe The New Frontiers-class Saturn Probe concept would deploy a probe into Saturn's atmosphere to measure its structure and composition (Visions 65 Voyages). While the materials used to construct such a probe may have improved since the construction of the Galileo probe (Young et al. 1996, and references within), the lifetime of the Saturn probe would still be limited, ultimately succumbing to the rising temperature and pressure as it descends. 
Comet and Interstellar Objects concepts During the last perihelion passage of Comet $1 / \mathrm{P}$ Halley, a figurative armada was sent to conduct close fly-bys of its nucleus. Because of the high relative velocities between the Earth and the comet, the durations of the fly-bys were short (no more than several hours near closest approach to its nucleus); the same would be true of any future mission to long-period comet. Inspired by both the success of the spacecraft that flew by Halley and the discovery of the first interstellar object (1I/2017 U1 'Oumuamua, Meech et al. 2017), there is active consideration of what kind of mission concept or concepts might be possible to enable a similar encounter with an interstellar object. While the relative velocities could be even higher than in the case of the Halley encounters, the potential science return from a close encounter with an interstellar object would be significant. Multiple white papers were submitted, both to the Science and Mission calls, describing the returns from encounters with either a long-period comet or interstellar object.

Radio \& Gravity Science The ability to precisely measure key properties of spacecraft radio signals - frequencies, phases, amplitudes, polarizations - has provided and will continue to provide unique leverage to extract information about planetary ionospheres, atmospheres, rings, surfaces, shapes, internal structure, and rotational dynamics. Radio Science or Gravity Science investigations have been a standard feature since the first interplanetary missions and are a portion of both current missions, e.g., Juno and BepiColombo (Asmar et al. 2017), and planned or likely for many future missions (e.g., Asmar et al. 2019, 2020).

Small Spacecraft Missions Multiple white papers have been submitted, either to the Science or the Mission call, describing the potential science return using small spacecraft. Stand-alone missions involving small spacecraft, such as under the Small Innovative Missions for Planetary Exploration (SIMPLEx) program, would likely require directto-Earth telecommunications, yet they can accommodate only smaller antennas and less powerful transmitters. Providing additional receiving capabilities on the ground may enable novel concepts, even beyond those described in the various white papers.

Interdisciplinary Science More broadly, NASA's Planetary Science missions can provide "ground truth" for NASA and NSF's Astronomy \& Astrophysics investigations, particularly for studies of extrasolar planets, which in turn provide context for the Solar System.

Finally, while our focus is on enabling larger data volumes to be returned, it is an unfortunate fact that spacecraft can enter a "safe mode." It is standard practice to include low-gain antennas on spacecraft for the purposes of enabling communications even if the spacecraft's attitude is unknown or uncontrolled. Low-gain antennas have the obvious benefit of a wide field of view at the cost of sensitivity. The received signal from a spacecraft in "safe mode" would be (much) weaker than planned from its high-gain antenna. During such times, having additional sensitive ground facilities can assist with recovery. 


\section{Collaborative Possibilities}

The primary benefit from collaborative work between NASA and NSF would occur in the acquisition of telemetry data (a.k.a. "downlink") from spacecraft beyond geosynchronous orbit. There are two key elements for assuring robust and large data volume returns: frequency coverage and sensitivity.

Table 1 summarizes the required frequency coverage. The standard deep space telecommunications frequency has been $\mathbf{X}$ band (approximately $8.5 \mathrm{GHz}$ ), but there is an increasing emphasis on missions having telecommunications systems at $\mathbf{K}$ band (approximately $26 \mathrm{GHz}$ ), in order to enable lunar missions and missions at the Earth-Sun Lagrange points, and in the $\mathbf{K a}$ band (approximately $32 \mathrm{GHz}$ ), for missions beyond the Lagrange points. These efforts are motivated by two increasingly problematic aspects of $\mathrm{X}$ band: (i) The international spectral allocations for spacecraft telecommunications provide narrower bandwidths, implying lower data rates, at X band as compared to K or Ka band. Specifically, only $50 \mathrm{MHz}$ of bandwidth is allocated at X band versus $1500 \mathrm{MHz}$ at both $\mathrm{K}$ and $\mathrm{Ka}$ band; and (ii) The number of missions using $\mathrm{X}$ band has grown over time to the point that spectral congestion can be an issue, i.e., interference between the transmissions from multiple spacecraft. This issue is particularly acute at Mars, where there are multiple spacecraft, from multiple space agencies.

There are also missions that make use of $\mathbf{S}$ band (approximately $2.3 \mathrm{GHz}$ ), which is useful for transmission through clouds (e.g., Venus), and the ultra-high frequency (UHF) portion (approximately $0.4 \mathrm{GHz}$ ) of the radio spectrum used for spacecraft-tospacecraft communications ("proximity links," e.g., surface of Mars to orbit or surface of Venus to orbit).

Table 2 illustrates (relative) sensitivities of relevant NASA and NSF facilities. The sensitivities shown are primarily a compar-

Table 1: Frequency Bands for Deep-Space Telemetry (Downlink)

\begin{tabular}{lcl}
\hline Band & $\begin{array}{c}\text { Frequency } \\
(\mathrm{GHz})\end{array}$ & Comment \\
\hline S band & $\begin{array}{l}2.20-2.29 \\
2.29-2.30\end{array}$ & $\begin{array}{l}\text { lunar, Lagrange points } \\
\text { beyond Lagrange points }\end{array}$ \\
& & \\
X band & $8.40-8.45$ & beyond Lagrange points \\
& $8.45-8.50$ & lunar, Lagrange points \\
& & \\
K band & $25.5-27.0$ & $\begin{array}{l}\text { lunar, Lagrange points } \\
\text { Ka band }\end{array}$ \\
\hline
\end{tabular}

ison of the relative collecting areas, as all facilities are (or would be) equipped with cryogenic microwave receivers and have (or would have) high antenna deficiencies. (Further, at $\mathrm{K}$ and $\mathrm{Ka}$ bands, both atmospheric conditions, e.g., the presence of clouds, and location of the target spacecraft [low on the horizon] can affect the sensitivity.)

NASA Deep Space Network The DSN consists of three Complexes (Goldstone, CA; Madrid, Spain; Canberra, Australia) distributed approximately at $120^{\circ}$ in longitude from each other in order to ensure essentially continuous coverage for a spacecraft 
Table 2: Telemetry Relative Performance Illustration

\begin{tabular}{|c|c|c|c|}
\hline \multirow[t]{2}{*}{ System } & \multicolumn{3}{|c|}{ Relative Performance } \\
\hline & $\mathrm{X}$ band & $\mathrm{K}$ band & Ka band \\
\hline NASA DSN $34 \mathrm{~m}$ & 1.0 & 7.9 & 11.8 \\
\hline NASA DSN $70 \mathrm{~m}$ & 4.3 & $\ldots$ & $\ldots$ \\
\hline NASA DSN $4 \times 34 \mathrm{~m}$ & 3.7 & 35.4 & 46.8 \\
\hline NSF Green Bank Telescope 100 m & 8.7 & 78.7 & 114 \\
\hline NSF Arecibo Observatory 300 m & 54.1 & $\ldots$ & $\ldots$ \\
\hline $\begin{array}{l}\text { NSF Very Large Array } \\
\text { (27 antennas, } 25 \mathrm{~m} \text { diameter })\end{array}$ & 14.6 & 115 & 172 \\
\hline $\begin{array}{l}\text { ngVLA Core Array } \\
\quad \text { (94 antennas, } 18 m \text { diameter) }\end{array}$ & 17.1 & 208 & 248 \\
\hline
\end{tabular}

anywhere in the Solar System. At each DSN Complex, there is a $70 \mathrm{~m}$ antenna and multiple $34 \mathrm{~m}$ antennas. Illustrated in Table 2 is the antenna arraying capability of the DSN, first introduced for the Voyager 2 encounters with Saturn, Uranus, and Neptune, which enables higher data volumes to be returned by virtual of the higher sensitivities obtained.

NSF Green Bank Telescope The $100 \mathrm{~m}$ diameter GBT has been used already in a collaborative manner with the Cassini-Huygens mission, as noted above. This telescope can operate at Ka-band frequencies (and higher), and it is fully steerable, offering access to spacecraft in nearly $75 \%$ of the sky.

NSF Arecibo Observatory The $300 \mathrm{~m}$ diameter Arecibo Observatory offers unmatched sensitivity at $\mathrm{S}$ band, and potential gains in sensitivity at $\mathrm{X}$ band for its current range of declinations $-1^{\circ}<\delta<+37.5^{\circ}$, which covers the ecliptic plane in the northern hemisphere. Higher frequency capability is not being considered at his time.

NSF Very Large Array The VLA has already been used in a collaborative manner with the DSN, for the Voyager 2 encounter at Neptune, as noted above. The antennas can operate at Ka-band frequencies (and higher) and can access spacecraft in $75 \%$ of the sky.

next-generation Very Large Array The ngVLA is a proposed telescope, consisting of up to 244 antennas of $18 \mathrm{~m}$ diameter, largely concentrated in the U.S. Southwest but with some antennas extending across North America (Selina et al. 2018). The antennas 
would be designed to operate at Ka-band frequencies (and higher) and would be fully steerable and therefore able to access as much as $75 \%$ of the sky. Table 2 shows the potential performance of the Core Array, which would consist of 94 antennas.

\section{Conclusion}

As is apparent, there are multiple potential opportunities for collaborative efforts between NASA and the NSF to enable significant data returns for future missions. The benefits will depend upon the details of a specific mission, and, in some scenarios, the data volume ultimately may be limited by other factors (e.g, whether a spacecraft bus could transfer data

from a science instrument to the telecommunications system). Nonetheless, the potential benefits for Planetary Science are likely to be rich and varied. 


\section{References}

Asmar, S. W., Bolton, S. J., Buccino, D. R., et al. 2017, "The Juno Gravity Science Instrument," Space Sci. Rev., 213, 205; doi: 10.1007/s11214-017-0428-7

Asmar, S. W., Lazio, J., Atkinson, D. H., Bell, D. J., Border, J. S., Grudinin, I. S., Mannucci, A. J., Paik, M., \& Preston, R. A. 2019, "Future of Planetary Atmospheric, Surface, and Interior Science Using Radio and Laser Links," Radio Sci., 54, 365; doi: 10.1029/2018RS006663

Asmar, S., et al. 2020, "Planetary Interiors, Atmospheres, and Surfaces Investigations via Radio Links: Goals for the Next Decade," Planetary Sciences Decadal Survey white paper

Folkner, W. M., Asmar, S. W. Border, J. S., Franklin, G. W., Finley, S. G., Gorelik, J., Johnston, D. V., Kerzhanovich, V. V., Lowe, S. T., Preston, R. A., Bird, M. K., DuttaRoy, R., Allison, M., Atkinson, D. H., Edenhofer, P., Plettemeier, D., \& Tyler, G. L. 2006, "Winds on Titan from ground-based tracking of the Huygens probe," J. Geophys. Res., 111, E07S02; doi: 10.1029/2005JE002649

Grundy, W. M., Bird, M. K., Britt, D. T., et al. 2020, "Color, composition, and thermal environment of Kuiper Belt object (486958) Arrokoth," Science, 367, 3705; doi: 10.1126/science.aay3705

Layland, J. W., \& Brown, D. W. 1985, "Planning for VLA/DSN Arrayed Support to the Voyager at Neptune," Telecommunications and Data Acquisition Report 42-82, Jet Propulsion Laboratory, California Institute of Technology

McKinnon, W. B., Richardson, D. C., Marohnic, J. C., et al. 2020, "The solar nebula origin of (486958) Arrokoth, a primordial contact binary in the Kuiper Belt," Science, 367, 6620; doi: 10.1126/science.aay6620

Meech, K. J., Weryk, R., Micheli, M., et al. 2017, "A brief visit from a red and extremely elongated interstellar asteroid," Nature, 552, 378; doi: 10.1038/nature25020

Rogstad, D. H., Mileant, A., \& Pham, T. T. 2003, Antenna Arraying Techniques in the Deep Space Network, Deep Space Communications and Navigation Series, Monograph 5, ed. J. H. Yuen (Jet Propulsion Laboratory, California Institute of Technology: Pasadena, CA) https://descanso.jpl.nasa.gov/monograph/series5/Descanso_Mono5_full.pdf

Selina, R. J., Murphy, E. J., McKinnon, M., et al. 2018, "The ngVLA Reference Design," in Science with a Next Generation Very Large Array, Astronomical Society of the Pacific Conference Series, vol. 517, ed. E. Murphy, (Astronomical Society of the Pacific: San Francisco, CA) p. 15; ISBN 978-1-58381-919-7

Spencer, J. R., Stern, S. A., Moore, J. M., et al. 2020, "The geology and geophysics of Kuiper Belt object (486958) Arrokoth," Science, 367, 3999; doi: 10.1126/science.aay3999

Young, R. E., Smith, M. A., \& Sobeck, C. K. 1996, "Galileo Probe: In situ Observations of Jupiter's Atmosphere," Science, 272, 837; doi: 10.1126/science.272.5263.837 